\title{
Le nouveau certificat de salaire et le cabinet médical
}

N. Reichen

Correspondance: FMH Services Fiduciaire Nicolas Reichen, juriste Fiduciaire Michel Favre SA Rue Pichard 20 CH-1003 Lausanne
D'un point de vue administratif, les cabinets médicaux peuvent pour la plupart être comparés à des petites et moyennes entreprises (PME), même lorsque ceux-ci présentent une taille modeste, par exemple au moment de l'installation du médecin à titre indépendant. En matières salariale et de personnel, dès le moment où le médecin emploie ne serait-ce qu'une assistante médicale, celui-ci se retrouve obligatoirement confronté aux différents problèmes de gestion rencontrés par tout entrepreneur, engendrant une charge additionnelle de travail.

Jusqu'à fin 2005, les certificats de salaire ne subissent pas de modification. Dès le $1^{\text {er }}$ janvier 2006, en principe, entre en vigueur le nouveau certificat de salaire, unifié à l'échelon national. Ce document, qui peut d'ores et déjà être utilisé cette année, est relativement technique. En effet, il a pour but d'annoncer clairement à l'autorité fiscale, respectivement aux entités sociales (notamment les administrations en charge de l'assurance vieillesse et survivants et de l'assurance chômage, ainsi que les fondations de prévoyance LPP), tous les revenus que l'employé perçoit, qu'ils soient versés en espèces ou en nature (repas offerts, véhicule d'entreprise mis à la disposition du salarié pour ses déplacements privés, allocations spéciales pour frais, par exemple).

A titre de rappel, avant de détailler les conséquences de l'introduction du nouveau certificat de salaire, il paraît utile de rappeler la notion d'une activité dépendante (salariée). Une activité salariée existe fiscalement lorsque la personne concernée «s'engage pour une durée déterminée ou indéterminée à fournir des prestations contre rémunération en se soumettant aux instructions de son employeur» (Arrêt du Tribunal Fédéral [ATF] 121 I 259).

La dénomination et/ou la forme des rétributions perçues par l'employé n'importe(nt) que peu, tant que la source des versements est un rapport de travail (article 17, alinéa premier, de la Loi sur l'impôt fédéral direct [LIFD]).

Dès lors, tout avantage perçu par l'employé en raison du contrat de travail, que celui-ci soit oral ou écrit, est considéré comme du salaire, sauf exceptions.
L'introduction du nouveau certificat de salaire aura une influence sur le contenu des «fiches de salaire» que les employeurs ont émis jusqu'ici. En effet, bien que la définition du revenu d'une activité lucrative dépendante n'ait pas été modifiée, il existera une obligation générale d'annoncer des prestations qui, jusqu'à ce jour, ne l'étaient pas nécessairement.

Dès l'entrée en vigueur obligatoire du nouveau certificat de salaire, les différents avantages susmentionnés devront faire l'objet d'une mention et de perceptions sociales potentiellement.

Vous trouverez à la fin de cet article l'illustration du nouveau certificat de salaire et, ci-après, les explications des différentes rubrique.

\section{Explications}

\section{Lettre A}

Cette case doit être cochée lorsque le formulaire sert d'établissement d'un certificat de salaire.

\section{Lettre B}

Cette case doit être cochée lorsque le formulaire sert d'établissement d'un certificat de rentes.

\section{Lettre C}

Compléter le champ avec le numéro d'AVS de l'employé et, si celui-ci n'est pas connu, remplir avec la date de naissance exacte.

\section{Lettre D}

Champ concernant l'année qui est couverte par le certificat de salaire.

\section{Lettre $\mathbf{E}$}

Période de travail dans l'année, même si l'employé est engagé pour toute l'année. En cas de rapports de travail sporadiques, spécifier la date des premier et dernier jours des rapports de travail dans l'année.

\section{Lettre $\mathbf{F}$}

Case à cocher si l'employé n'engage pas de frais entre son lieu de domicile et celui de travail, notamment quand une voiture est mise à sa dis- 
position par l'employeur, quand l'abonnement des transports publics est payé, même partiellement, par celui-ci ou quand l'employeur indemnise son employé pour se rendre au travail au moyen de son véhicule privé.

\section{Lettre G}

Case à cocher si l'employé a la possibilité de se restaurer dans une cantine et/ou s'il peut bénéficier de réductions pour ses repas pris au restaurant (par exemple bons de repas remis par l'employeur ou vendus à un tarif préférentiel).

\section{Lettre $\mathbf{H}$}

Coordonnées actuelles de l'employé (nom, prénom, adresse).

\section{Lettre I}

Lieu et date, coordonnées de l'entreprise et signature valable (si le certificat n'est pas établi de manière informatique).

\section{Chiffre 1}

Salaire ou rente: il faut faire figurer sous cette rubrique:

- le salaire ordinaire;

- toutes les allocations touchées par le collaborateur, notamment allocations de naissance, allocations familiales, allocations pour travail par équipe, allocations pour travail de nuit, etc. Dans le cas où certaines allocations devraient être versées par une Caisse de compensation, il y a lieu de le mentionner clairement sous chiffre 15 (Observations);

- les commissions;

- les indemnités de trajet autres que celles concernées par la case «F»;

- toutes contributions touchées au comptant pour les repas pris au lieu de travail, sauf dans le cas de chèques repas si le montant n'excède par Fr. 180.- par mois (limite AVS au

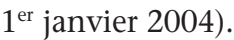

\section{Chiffre 2}

Prestations en nature touchées par le collaborateur à leur valeur vénale ou marchande (cf. Notice N2/2001 de l'AFC):

- chiffre 2.1: la pension et le logement fournis à titre gratuit au collaborateur. Si le logement est constitué d'un appartement et non d'une chambre, il y a lieu de le préciser sous chiffre 2.3;

- chiffre 2.2: déterminer l'avantage en francs que perçoit le collaborateur pour l'utilisation privée d'un véhicule de l'entreprise. Si tous les frais courants sont en plus à la charge de l'employeur, on comptera $1 \%$ de la valeur vé- nale (valeur d'acquisition) du véhicule à titre de prestation mensuelle fournie au collaborateur;

- chiffre 2.3: toutes les autres prestations en nature fournies au collaborateur, notamment abonnement général CFF pour les trajets domicile - lieu de travail, les appartements de fonction, etc.

\section{Chiffre 3}

Prestations non périodiques versées au collaborateur telles que bonus (versés en fonction de la prestation effectuée), prime d'engagement, prime de fidélité, prime d'ancienneté de service, indemnités de déménagement.

\section{Chiffre 4}

Prestations en capital versées au collaborateur (nature à spécifier sur la ligne correspondante pour déterminer le taux d'imposition). Elles peuvent notamment être des indemnités de départ, des prestations en capital à caractère de prévoyance et des versements différés de salaire (heures supplémentaires).

\section{Chiffre 5}

Droits de participation. La valeur du droit est égale à la différence entre la valeur vénale de la participation (éventuellement escomptée) et le prix de cession ou d'acquisition.

\section{Chiffre 6}

Toutes les indemnités de membres de l'administration versées au collaborateur, en particulier les indemnités de membres de conseil d'administration, les jetons de présence et les tantièmes.

\section{Chiffre 7}

Toutes les autres prestations (à spécifier clairement dans les champs prévus à cet effet) soit:

- les indemnités journalières d'assurance;

- les prestations de l'assurance chômage;

- les allocations pour perte de gain (APG);

- les prestations prises en charge par l'employeur pour le compte de l'employé pour l'AVS/AI/APG/AC, pour l'AANP et pour le $2^{\mathrm{e}}$ pilier;

- toutes les cotisations de l'employeur à des assurances privées de l'employé;

- l'impôt à la source pris en charge par l'employeur.

\section{Chiffre 8}

Salaire brut total (addition des chiffres 1 à 7).

\section{Chiffre 9}

Cotisations AVS/AI/APG/AC. 


\section{Chiffre 10}

Déductions relatives aux cotisations à des institutions de prévoyance professionnelle collective ( $2^{\mathrm{e}}$ pilier):

- cotisations ordinaires sous chiffre 10.1 versées selon les dispositions légales;

- cotisations pour le rachat dans la prévoyance professionnelle sous chiffre 10.2 soit, des cotisations de rachat à la suite d'une augmentation de salaire, des cotisations de rachat d'années d'assurance manquantes, des cotisations pour un rachat nécessité par une modification du règlement ou du plan de prévoyance ou des cotisations de rachat en cas de divorce.

\section{Chiffre 11}

Salaire net (chiffres 8, 9 et 10).

\section{Chiffre 12}

Retenues concernant l'impôt à la source pour tous les collaborateurs qui ne sont pas suisses ou qui sont au bénéfice d'un permis d'établissement «C» (pour les cas particuliers, se référer aux normes concernant l'impôt à la source).

\section{Chiffre 13}

Ce point comprend toutes les autres indemnités ou prestations versées par l'employeur ne figurant pas dans les points précédents, regroupées sous le terme «allocations pour frais». Les allocations pour frais sont des indemnités versées au collaborateur pour les dépenses qu'il a engagées à l'occasion d'une mission professionnelle concrète.

Les allocations forfaitaires pour frais sont toutes les indemnités versées pour une période déterminée indépendamment des frais engagés.

Les allocations effectives pour frais comportent toutes les indemnités qui ne sont pas versées régulièrement, mais à chaque fois que des frais sont engagés. Ces frais ne doivent pas être mentionnés, sauf s'ils dépassent les limites suivantes:

- frais de nuitées contre justificatifs;

- frais de repas pour un montant de Fr. 35.maximum par repas; si forfait: Fr. 30.- au maximum;

- les invitations des clients doivent correspondre aux décomptes TVA;

- l'utilisation des transports publics (train/ avion) doit être prouvée par des justificatifs;

- un maximum de Fr. -.70 est versé pour l'utilisation du véhicule privé à titre professionnel;

- les menus frais peuvent être remboursés forfaitairement jusqu'à concurrence de Fr. 20.par jour.
Les frais sont répartis sous divers points de manière suivante:

- chiffre 13.1.1: tous les frais effectifs de voyage, de repas et de nuitées tels que les frais effectifs de voiture, d'avion, de taxi et de train, les frais de déjeuners, dîners et soupers, les frais d'invitation des partenaires commerciaux au restaurant ou chez soi, les frais de collations prises pendant le trajet, les frais d'hôtel et de cadeaux offerts en cas de logement chez des particuliers;

- chiffre 13.1.2: les autres frais effectifs tels que les indemnités pour les dépenses professionnelles particulières des expatriés, les menues dépenses engagées durant un déplacement professionnel, les frais de visa, de certificats de vaccination, etc.;

- chiffre 13.2.1: les frais de représentation forfaitaires versés au collaborateur pour ses menues dépenses;

- chiffre 13.2.2: comprend les frais de véhicule versés au collaborateur pour l'utilisation de son véhicule privé à des fins professionnelles (en général plus de 10000 km par an);

- chiffre 13.2.3: les autres frais forfaitaires qui ne répondent pas exactement aux définitions des frais de représentation et des frais de véhicule;

- chiffre 13.3: toutes les indemnités versées en espèces au collaborateur pour des cours de perfectionnement (y compris trajets et repas); englobe également toute contribution versée à un tiers pour des cours dépassant Fr. 10 000.- par an et par employé.

\section{Chiffre 14}

Les prestations salariales accessoires sont les avantages appréciables en argent que l'employeur peut fournir à son collaborateur à des conditions favorables, notamment des prix préférentiels sur des services.

\section{Chiffre 15}

Les observations complémentaires comportent toutes les indications qui ne peuvent figurer dans les autres rubriques et qui sont indispensables à l'exactitude et à la clarté du certificat de salaire, soit:

- nombre de jours ayant donné lieu au versement d'indemnités pour perte de gain et éventuellement spécifier si ce n'est pas compris dans le salaire brut;

- tout salaire supplémentaire versé de l'étranger;

- l'agrément d'un règlement relatif aux frais (par exemple canton de Vaud);

- la délivrance de plusieurs certificats de salaire; 
- degré d'occupation du collaborateur si celuici ne travaille pas à $100 \%$;

- participation octroyée au collaborateur;

- indemnités supplémentaires d'une tierce entreprise.

Il faut noter que pour les collaborateurs au bénéfice de frais forfaitaires, l'entreprise doit déposer un règlement d'entreprise à l'Administration Cantonale des Impôts en mentionnant les bénéficiaires et les montants alloués.

En cas d'inobservation de ces règles par l'employeur, ce dernier s'expose à des amendes pouvant aller jusqu'à Fr. 50 000.- fixées en fonction de la faute commise.

Si le nouveau certificat de salaire est, par ses exigences de précision, constitutif d'un surcroît de travail administratif pour l'employeur - et donc le médecin exploitant un cabinet médical -, il a le mérite de clarifier la situation en matière de revenu imposable et soumis aux charges sociales.

En revanche, lorsqu'un employeur annoncera tous les avantages que l'employé obtient en vertu de son contrat de travail, il pourrait se retrouver dans une situation délicate par rapport à l'autorité fiscale, lorsque des revenus jusqu'ici non déclarés apparaissent dans le nouveau certificat de salaire. Des rattrapages, voire des amendes, pourraient être engendrés par une telle situation avec des conséquences comparables en matière de perceptions sociales notamment.

Il serait judicieux pour les employeurs de préparer la transition pour l'entrée en vigueur obligatoire de ce nouveau formulaire dans les délais les plus brefs en prenant contact avec leur conseil en matière comptable et fiscale en vue de la transition qui devra être opérée en principe au premier janvier 2005.

Tableau 1

Implications des versements complémentaires pour les impôts directs, I'AVS et le $2^{\mathrm{e}}$ pilier.

\begin{tabular}{|c|c|c|c|c|}
\hline $\mathbf{N}^{\circ}$ & Description de la prestation & Soumis Impôts & Soumis AVS & Soumis LPP \\
\hline 2.1 & Pension et logement & oui & oui & oui \\
\hline 2.2 & Part privée voiture de service & oui & oui & oui \\
\hline 2.3 & Autres prestations en nature & oui & oui & oui (si régulières) \\
\hline 3 & Prestations non périodiques & oui & de cas en cas & non (sauf bonus) \\
\hline 4 & Prestations en capital & oui (taux réduit) & de cas en cas & non (caractère unique) \\
\hline 5 & Droits de participation & oui & oui & non (si pas soumis AVS) \\
\hline 6 & Indemnités membres administration & oui & oui & non (caractère de gains accessoires) \\
\hline 7 & Autres prestations & oui & oui & non (si pas soumis AVS)* \\
\hline 13.1 .1 & Voyages, frais de repas, nuitées (effectifs) & non & non & non (si pas soumis AVS) \\
\hline 13.1 .2 & Autres frais (effectifs) & non & non & non (si pas soumis AVS) \\
\hline 13.2 .1 & Frais de représentation (forfaitaires) & non (si règlement) & oui & non (si pas soumis AVS)* \\
\hline 13.2 .2 & Frais de voiture (forfaitaires) & non (si règlement) & oui & non (si pas soumis AVS)* \\
\hline 13.2 .3 & Autres frais (forfaitaires) & non (si règlement) & oui & non (si pas soumis AVS)* \\
\hline
\end{tabular}


A Lohnausweis - Certificat de salaire - Certificato di salario

B Rentenbescheinigung - Attestation de rentes - Attestazione delle rendite

C

AHV-Nr. - No AVS - N. AVS

D

(D)

Jahr - Année - Anno

von - du - dal

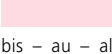

Unentgeltliche Beförderung zwischen Wohn- und Arbeitsort

Transport gratuit entre le domicile et le lieu de travail

Trasporto gratuito dat domicilio al luogoc

G Kantinenverpflegung/Lunch-Checks

Repas à la cantine / chèques-repas

Pasti alla mensa / buoni pasto

H

1. Lohn soweit nicht unter Ziffer $2-7$ aufzuführen / Rente Salaire qui ne concerne pas les chiffres 2 à 7 ci-dessous / Rente Salario se non da indicare sotto cifre da 2 a 7 più sotto / Rendita

2. Gehaltsnebenleistungen $\mathbf{2 . 1}$ Verpflegung, Unterkunft - Pension, logement - Vitto, alloggio Prestations salariales accessoires

Prestazioni accessorie al salario 2.2 Privatanteil Geschäftswagen - Part privée voiture de service - Quota privata automobile di servizio +

2.3 Andere - Autres - Altre Art - Genre - Genere

3. Unregelmässige Leistungen - Prestations non périodiques - Prestazioni aperiodiche Art - Genre - Genere

4. Kapitalleistungen - Prestations en capital - Prestazioni in capitale Art - Genre - Genere

5. Beteiligungsrechte gemäss Beiblatt - Droits de participation selon annexe - Diritti di partecipazione secondo allegato

6. Verwaltungsratsentschädigungen - Indemnités des membres de l'administration - Indennità dei membri di consigli d'amministrazione

7. Andere Leistungen Art

Autres prestations Genre

Altre prestazioni Genere

8. Bruttolohn total / Rente - Salaire brut total / Rente - Salario lordo totale / Rendita

9. Beiträge AHV/IV/EO/ALV/NBUV - Cotisations AVS/AI/APG/AC/AANP - Contributi AVS/AI/IPG/AD/AINP

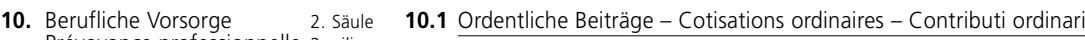
Prévoyance professionnelle $2^{\mathrm{e}}$ pilier

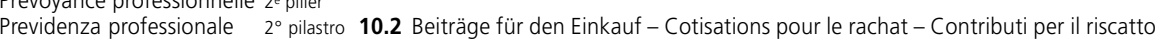

11. Nettolohn / Rente - Salaire net / Rente - Salario netto / Rendita In die Steuererklärung übertragen - A reporter sur la déclaration d'impôt - Da riportare nella dichiarazione d'imposta

12. Quellensteuerabzug - Retenue de l'impôt à la source - Ritenuta d'imposta alla fonte

13. Spesenvergütungen - Allocations pour frais - Indennità per spese

Nicht im Bruttolohn (gemäss Ziffer 8) enthalten - Non comprises dans le salaire brut (au chiffre 8) - Non comprese nel salario lordo (sotto cifra 8)

13.1 Effektive Spesen 13.1.1 Reise, Verpflegung, Übernachtung - Voyage, repas, nuitées - Viaggio, vitto, alloggio

Frais effectifs $\quad$ 13.1.2 Übrige - Autres - Altre

Spese effettive Art-Genre-Gener

13.2 Pauschalspesen 13.2.1 Repräsentation - Représentation - Rappresentanza

Frais forfaitaires

Spese forfettarie 13.2.2 Auto - Voiture - Automobile

13.2.3 Übrige - Autres - Altre
Art - Genre - Genere

13.3 Beiträge an die Weiterbildung - Contributions au perfectionnement - Contributi per il perfezionamento

14. Weitere Gehaltsnebenleistungen Art

Autres prestations salariales accessoires Genre

Altre prestazioni accessorie al salario Genere

15. Bemerkungen

Observations

Osservazioni

I Ort und Datum - Lieu et date - Luogo e data Die Richtigkeit und Vollständigkeit bestätigt

inkl. genauer Anschrift und Telefonnummer des Arbeitgebers

Certifié exact et complet

y.c. adresse et numéro de téléphone exacts de l'employeur

Certificato esatto e completo

compresi indirizzo e numero di telefono esatti del datore di lavoro

Form 11-2005-dfi $\quad(15.9 .2004)$ 